\title{
Patellar Tendon Quality and Knee Symptoms in Male Athletes Before the Anterior Cruciate Ligament Reconstruction
}

\section{Carla Soncino Pereira ( $\nabla$ carla.pereira@aspetar.com )}

Aspetar Orthopaedic and Sports Medicine Hospital https://orcid.org/0000-0003-2920-1300

\section{Jasenko Klauznicer}

Aspetar Orthopaedic and Sports Medicine Hospital Rehabilitation Department

\section{Sean McAuliffe}

Qatar University

\section{Rodney Whiteley}

Aspetar Orthopaedic and Sports Medicine Hospital Rehabilitation Department

Taija Juutinen Finni

University of Jyväskylä Faculty of Sports Science: Jyvaskylan yliopisto Liikuntatieteellinen tiedekunta

\section{Research article}

Keywords: anterior cruciate ligament, anterior knee pain, echo-types distribution, patellar tendon, tendon quality, UTC

Posted Date: December 22nd, 2020

DOI: https://doi.org/10.21203/rs.3.rs-131706/v1

License: @ (i) This work is licensed under a Creative Commons Attribution 4.0 International License. Read Full License 


\section{Abstract}

Background: It is unknown if the quality of the patellar tendon is affected by an anterior cruciate ligament (ACL) injury. ACL deficient patients (ACL-D) have shown to have knee pain, quadriceps weakness and reduced knee function. Ultrasound tissue characterization (UTC) has been used to assess the quality of tendons by quantifying the proportion of echo-types using a numerical grading system from best to worst (I-IV). The primary aims of this cross-sectional study were to investigate the patellar tendon quality in athletes with unilateral ACL injuries, and to compare them to asymptomatic control tendons. The secondary aims were to assess the impact of anterior knee pain (AKP) in the patellar tendons of ACL$D$, and to explore possible correlations between tendon quality and knee pain, knee extensor strength and time from $A C L$ injury.

Methods: UTC was used to scan both patellar tendons of 81 ACL-D athletes and 20 control tendons. Echo-types distribution was calculated to compare the quality between limbs of ACL-D and control tendons; and to compare ACL-D with AKP, other knee pain, and without pain. Associations between the variables of tendon quality and knee symptoms were explored.

Results: No difference in tendon quality was found between limbs of ACL-D. Both tendons of ACL-D displayed more echotype II at the patellar apex, and same or less echo-types III and IV than controls. The proximal half of the control tendons displayed better quality than the distal half. ACL-D knees with pain displayed less extensor strength, however, in addition to the lack of difference in tendon quality regardless of the presence and location of pain, there was no meaningful association between pain, extensor strength and tendon quality. Nevertheless, there were weak to moderate associations between tendon quality and time from ACL injury.

Conclusion: Despite the ACL injury, there is no difference in tendon quality between ACL-D limbs, or when subgrouping ACL$\mathrm{D}$ tendons by presence and location of knee pain. Different areas of the patellar tendon may present different characteristics that might be related to training. Tendon quality is better if a person has longer time from injury.

\section{Background}

Anterior cruciate ligament (ACL) injuries are a significant cause of time loss in sport [1, 2]. Frequently, ACL deficient (ACL-D) patients present with a restricted range of motion (ROM) and pain in the acute phase [3]. The pain intensity and location varies depending on the presence and severity of the associated injuries such as meniscal lesions and collateral ligament involvement [4]. In particular, anterior knee pain (AKP) commonly occurs in ACL-D patients, possibly due to the aggressive mechanism of the ACL injury itself [5], the initial restricted joint mobility [3], pre-existing patella-femoral joint disorders [6] and/or patellar tendon abnormalities [6]. Athletes involved in jumping sports such as volleyball, handball, and basketball may often have a pre-injury history of AKP, most likely attributed to patellar tendon pain [7]. It may be postulated that an $A C L$ injury followed by an ACL reconstruction may aggravate this problem. AKP is also commonly present after $A C L$ reconstruction [8], which might be influenced by the graft choice, and might lead to persistent knee extensor muscles weakness [8-11]. It has been proposed that less AKP before the ACL reconstruction is predictive of better clinical outcomes at 12 months post-operative $[12,13]$. Concurrently, preoperative quadriceps strength deficits greater than $20 \%$ in comparison to the uninvolved limb are related to long lasting strength deficits after the ACL reconstruction [8].

One method of exploring AKP is the use of ultrasound tissue characterization imaging (UTC), which is a valid and reliable tool to assess the quality of healthy [14-16], pathological [14-16], and harvested tendons [17]. It categorizes the tendon structure based on the stability of the echo-pattern and quantifies the percentage of four distinct echo-types: I to IV; from more aligned (type I) to least organized (type IV) tendon structure $[14,18,19]$. UTC has been increasingly used to quantify the effects of training load in patellar and Achilles tendons. It has been proposed that excessive loading might alter tendon homeostasis leading to collagen degradation with associated swelling and/or increased waviness of the tendon bundles. These adaptations could cause a reduction in echo-type I and/or an increase in echo-type II in the UTC scans that may or may not be associated to AKP $[20,21]$. Conversely, there is some evidence to suggest that targeted loading improves the 
tolerance of the tendon, resulting in more aligned tendon tissue, which in UTC scans could be represented by an increase in echo-type I with associated reduction in echo-type II [22].

To the best of our knowledge, it is unknown whether ACL injuries have an impact on the quality of patellar tendons in ACL$D$ athletes. Considering AKP is often present in ACL-D patients, it seems plausible to suggest that one potential reason for AKP may be attributed to alterations in the patellar tendon quality. Given the initial period of rest and joint protection following an ACL injury, the de-loading, and the subsequent rapid attempts of re-loading the lower limb may alter the patellar tendon capacity. Thus, understanding the nature and potential impact of AKP in the patellar tendon's structure and knee extensor muscles of athletes who have sustained a unilateral ACL injury may have important implications for rehabilitation. To be able to differentiate the source of pain could limit the deleterious effects of AKP and allow targeted interventions to improve treatment outcomes.

Therefore, the aim of the present study was to investigate the effect of a unilateral ACL injury on the quality of both patellar tendons, and to explore the possible associations between patellar tendon structure with knee pain, knee extensor muscle strength and the time from the ACL injury. We hypothesized that the involved patellar tendon of the ACL-D participants will display lower quality on the UTC scan in comparison to the uninvolved patellar tendon, and that both patellar tendons of the ACL-D participants will present lower quality on the UTC scan in comparison to a matching control group. Furthermore, we expected that ACL-D participants with AKP would present lower quality of the symptomatic patellar tendon in comparison to participants with pain in different areas of the involved knee and in comparison, to the tendons of asymptomatic knees.

\section{Methods}

\section{Participants}

Four hundred and twenty-five male professional athletes registered at any sporting club or federation within Qatar were eligible for inclusion in the study. Eligible participants were examined in the assessment center of Aspetar, Orthopedic and Sports Medicine Hospital from July 2015 to March 2020. Participants were eligible to take part in this study if they were: male, aged between 18 to 45 years, had a unilateral ACL rupture detected by clinical tests (Lachman and Pivot Shift) [23, 24] which was confirmed by magnetic resonance imaging, referred to start or had recently enrolled in the ACL rehabilitation program of Aspetar. Participants were excluded if they presented with bilateral or previous ACL reconstruction in the contralateral limb, were 17 years or younger, or were unable to flex the affected knee sufficiently to achieve the required position for appropriate imaging of the patellar tendon. Participants in the control group were recruited from a cohort of professional football players participating in the Qatar Stars League and Qatar Gas League as part of a mid-season medical screening at the same venue. Included participants in the control group had no recent history of lower limb pathology, no previous ACL injury, or symptoms in their knees. The healthy active control group matched the studied ACL-D group in age, height, and current body mass. Since there was no significant difference between the right and left tendons of the 11 asymptomatic matched participants in the control group (Additional file 1), these were pooled for subsequent analyses. Note however, the UTC scans of two of these tendons did not meet the criteria of good alignment, leaving 20 patellar tendons for the comparative analysis between ACL-D and control tendons. Figure 1 shows the flow chart of the participant inclusion and exclusion. Informed consent was obtained from each participant. The study protocol was approved by the ethical committee of the Anti-Doping Laboratory Qatar Research Office (2017000227).

\section{Clinical Assessment}

Participant medical history was recorded, as well as participant demographics, including age, height, current body mass, and date of injury. Participants were asked specifically about the presence of knee pain. Pain location was determined 
based on participants' statement, and a 0-10 visual analogue scale (VAS) [25] was used to obtain the subjective score of maximum knee pain in the last 7 days prior to the assessment. The number of days between the date of the ACL injury and the date of data acquisition was calculated.

\section{Ultrasound Tissue Characterization (UTC) analysis and processing}

The patellar tendon quality was assessed using UTC. This method utilizes a 5-12 MHz ultrasound transducer (SmartProbe 12L5, Terason 2000, Teratech, USA) fixed into a tracking device (UTC Tracker, UTC imaging, Netherlands). About 600 sequential transverse images of the anterior knee were acquired from proximal to distal at regular intervals of $0.2 \mathrm{~mm}$. Each subject lay supine on the plinth with their knees flexed to approximately $100^{\circ}$. The UTC tracker was placed in parallel to the long axis of the patellar tendon resting with full contact on the anterior surface of the knee. Ultrasound parameters were standardized as: $12 \mathrm{MHz}$, with focus at $2.8 \mathrm{~cm}$ and depth of $4 \mathrm{~cm}$. Only scans that presented a horizontal and taut patellar tendon in the sagittal view (FIGURE 2A), and with the patellar apex and tibial tuberosity aligned longitudinally in the coronal view were included for analysis (FIGURE 2B).

UTC analyses were performed on the UTC analyzer v.2.0.2 with window size 17. In the transverse view of the UTC analyzer several contours were manually traced with a maximum of $0.5 \mathrm{~cm}$ apart (Fig. $3 \mathrm{~A}$ ) along the patellar tendon length for each tendon (Fig. 3B). The main areas of interest were: (1) apex (the first image after the patellar apex disappears in the transverse view); (2) proximal tendon (0.5 cm distal to the apex); (3) mid tendon (1.5 cm distal to the apex); and (4) distal tendon (at $75 \%$ of the tendon length) (FIGURE 3B). The UTC algorithm yielded the proportion of echo-types I (green aligned collagen bundles), II (blue - wavy collagen bundles), III (red - loose matrix), and IV (black - mainly amorphous matrix) in each area of interest. $[14,18]$ Detailed protocol for the patellar tendon data acquisition, analysis of the intra- and inter-rater reliability as well as the minimal detectable changes (MDC) for patellar tendons of ACL-D athletes have been previously described. [17]

(A) Transverse view of UTC color coded image showing a manually traced contour to delineate the area of the patellar tendon. (B) Sagittal view of the patellar tendon illustrating all the contours traced in the whole length of the patellar tendon. Yellow arrows identify the four levels of the tendon (1-4) where the proportion of echo-types were calculated to subcharacterize the tendon quality. (1) Patellar apex = first image after the patellar apex disappears in the transverse view. (2) Proximal tendon $=$ at $0.5 \mathrm{~cm}$ from patellar apex. (3) Mid tendon $=$ at $1.5 \mathrm{~cm}$ from patellar apex. (4) Distal tendon $=$ at $75 \%$ of the tendon length.

\section{Isokinetic strength test}

A Biodex ${ }^{\text {TM }}$ dynamometer (Biodex ${ }^{\text {TM }}$, Shirley, New York) was used for the evaluation of concentric peak torque of both uninvolved and involved lower limbs, in this order. After warming up on a stationary bicycle for approximately 10 minutes participants were seated upright with $90^{\circ}$ of hip and knee flexion. After a brief familiarization, the participants were asked to perform five repetitions of maximal knee extension and flexion for each lower limb at $60 \% \mathrm{~s}$ [24]. Concentric peak torque for knee extension $(\mathrm{Nm})$ as percentage of the body weight $(\mathrm{Kg})$ was recorded for each respective limb.

\section{Statistical Analysis}

Data distribution for normality was tested using the Shapiro-Wilk test. The variable of knee extensor strength presented normal pattern of distribution, therefore was analyzed with parametric tests. All the remaining studied variables were analyzed with non-parametric tests.

Descriptive statistics such as means, standard deviations, medians, interquartile ranges, minimums, and maximums were calculated for all variables of interest and are presented where applicable. 
To compare right and left tendons and the involved and uninvolved lower limbs, paired t-test and Wilcoxon signed ranks test were used for parametric and non-parametric variables, respectively.

As appropriate either a parametric independent samples t-test with equal variances, or the non-parametric equivalent MannWhitney $\mathrm{U}$ test for 2 independent samples was used for comparisons between ACL-D and controls. For the comparisons among the group of ACL-D participants with AKP, other knee pain, and without pain, the non-parametric Kruskal-Wallis test for $\mathrm{K}$ independent samples was used.

Due to the different sample sizes of ACL-D and control groups, and the non-parametric nature of the studied variables Hedge's coefficient $(g)$ was used to estimate the effect sizes (ES), ( $g=\left(\right.$ Mean $_{\text {ACL-D }}-$ Mean $\left._{\text {Control }}\right) / \sqrt{ }\left[\left(\mathrm{SD}_{\text {Control }^{2}}+\right.\right.$ $\left.\left.\left.S_{A C L-D}^{2}\right) / 2\right]\right)$. Thresholds for small, medium, and large effect sizes were $0.2,0.5$, and 0.8 , respectively [26].

Spearman's rank-order correlation was used to assess the strength and direction of the association between tendon quality and the variables of: VAS, knee extensor strength as percentage of the body, and time from ACL injury.

Statistical significance was set as $P<0.05$. SPSS v.24 was used for all statistical analyses (SPSS Inc, Chicago, Illinois, USA).

\section{Results}

The characteristics of the 81 included ACL-D participants and the 11 matching controls at the time of data acquisition are detailed in Table 1. While all the participants from the control group were active professional football players, the participants from ACL-D group were involved in a variety of sports. Of the ACL-D participants, 38 were football players, 9 were engaged in handball, 6 in basketball, 6 in rugby, 5 in futsal, 5 in volleyball, 2 in field hockey, and 1 in each of the following: athletics, beach soccer, billiards, cycling, rowing, sky diving, swimming, table tennis, tennis, and wrestling.

Fifty-three ACL-D participants had pain somewhere in the involved knee, whilst 12 of them had AKP specifically. The UTC scans revealed that a total of 23 tendons from 16 ACL-D participants displayed a clear disorganized area within the patellar tendon, namely, increased proximal thickness and proximal or distal darker areas. Seven of these participants presented one or more of these features in both patellar tendons, 5/16 in the involved tendon and 4/16 in the uninvolved tendon. From these athletes, 8 were engaged in football, 3 in basketball, 2 in rugby, 1 in volleyball, 1 in handball, and 1 in field hockey. While only 2 of these 23 tendons were associated with AKP at the time of data acquisition and another 9 participants presented some sort of knee pain, 5 of them had a pre-existent history of tendinopathy. 
Table 1

Descriptive statistics of the characteristics of ACL-D and control groups.

\begin{tabular}{|c|c|c|c|c|c|c|c|c|}
\hline & \multicolumn{3}{|c|}{ ACL-D $(n=81)$} & \multicolumn{5}{|c|}{ Control Group $(n=11)$} \\
\hline & $\begin{array}{l}\text { Mean } \pm \\
\text { SD }\end{array}$ & Median [IQR] & $\begin{array}{l}\text { Min - } \\
\text { Max }\end{array}$ & $\begin{array}{l}\text { Mean } \pm \\
\text { SD }\end{array}$ & Median [IQR] & $\begin{array}{l}\text { Min - } \\
\text { Max }\end{array}$ & $\begin{array}{l}\mathrm{p} \text { - } \\
\text { value }\end{array}$ & $\begin{array}{l}\text { Effect } \\
\text { size }\end{array}$ \\
\hline BW (kg) & $\begin{array}{l}79.5 \pm \\
14.5\end{array}$ & $\begin{array}{l}80.0 \text { [69.3- } \\
86.6]\end{array}$ & $\begin{array}{l}48.7- \\
122.5\end{array}$ & $\begin{array}{l}73.0 \pm \\
6.7\end{array}$ & $\begin{array}{l}73.8[69.4- \\
76.6]\end{array}$ & $\begin{array}{l}62.6- \\
85.8\end{array}$ & 0.09 & 0.47 \\
\hline Height $(m)^{\dagger}$ & $\begin{array}{l}1.78 \pm \\
0.09\end{array}$ & $\begin{array}{l}1.78[1.73- \\
1.84]\end{array}$ & $\begin{array}{l}1.55- \\
2.03\end{array}$ & $\begin{array}{l}1.77 \pm \\
0.07\end{array}$ & $\begin{array}{l}1.79 \text { [1.72- } \\
1.82]\end{array}$ & $\begin{array}{l}1.66- \\
1.86\end{array}$ & 0.78 & 0.11 \\
\hline Age (years) & $25 \pm 5$ & 24 [21-27] & $18-44$ & $\begin{array}{l}26.7 \pm \\
5.7\end{array}$ & $26[22-30]$ & $19-36$ & 0.14 & 0.33 \\
\hline \multirow{3}{*}{$\begin{array}{l}\text { Days from ACL } \\
\text { injury }\end{array}$} & $61 \pm 92$ & $33[20-61]$ & $6-622$ & - & - & - & - & - \\
\hline & \multicolumn{3}{|c|}{ ACL-D Involved $(n=69)$} & \multicolumn{5}{|c|}{ ACL-D Uninvolved $(n=74)$} \\
\hline & $\begin{array}{l}\text { Mean } \pm \\
\text { SD }\end{array}$ & Median [IQR] & $\begin{array}{l}\text { Min - } \\
\text { Max }\end{array}$ & $\begin{array}{l}\text { Mean } \pm \\
\text { SD }\end{array}$ & Median [IQR] & $\begin{array}{l}\text { Min - } \\
\text { Max }\end{array}$ & $\begin{array}{l}\mathrm{p}- \\
\text { value }\end{array}$ & $\begin{array}{l}\text { Effect } \\
\text { size }\end{array}$ \\
\hline $\begin{array}{l}\text { Ext PT\%BW } \\
(\%)^{\dagger}\end{array}$ & $\begin{array}{l}227.1 \pm \\
65.1 *\end{array}$ & $\begin{array}{l}227.6[183.7- \\
267.1]\end{array}$ & $\begin{array}{l}55.9- \\
371.0\end{array}$ & $\begin{array}{l}287.3 \pm \\
54.5 \text { * }\end{array}$ & $\begin{array}{l}286.3[254.5- \\
325.1]\end{array}$ & $\begin{array}{l}163.9- \\
450.2\end{array}$ & $<.01$ & 0.96 \\
\hline VAS $(0-10)$ & $2.4 \pm 2.1$ & $2.0[0.0-4.0]$ & $0.0-8.0$ & 0 & 0 & 0 & $\begin{array}{l}< \\
0.01\end{array}$ & 1.61 \\
\hline
\end{tabular}

$\mathrm{SD}=$ standard deviation.

IQR = interquartile.

BW = body weight.

VAS = Visual analogue scale

Ext PT\%BW = maximal knee extensor's peak torque as percentage of body weight.

${ }^{\dagger}$ Variable with normal pattern of distribution.

* Difference with statistical significance.

Comparisons between involved and uninvolved limbs of ACL-D participants displayed no significant difference in echotypes distribution $(P>0.05)$ (Table 2; FIGURE 4). The lack of difference in patellar tendon quality between limbs of ACL-D group was despite the fact that 53 ACL-D participants presented pain somewhere in the involved knee $(E S=1.61 ; P<0.01)$, and generated significant lower knee extensor strength than the uninvolved limb $(E S=0.96 ; P<0.01)($ Table 1$)$. 
Table 2

Echo-type distribution (\%) in the involved and uninvolved tendons of ACL-D and controls.

\begin{tabular}{|c|c|c|c|c|c|c|c|c|c|c|c|}
\hline \multirow{3}{*}{$\begin{array}{l}\text { Tendon } \\
\text { quality } \\
\text { (\%) }\end{array}$} & \multirow{2}{*}{\multicolumn{2}{|c|}{ Control $(n=20)$}} & \multicolumn{2}{|c|}{ ACL-D $(n=81)$} & & \multicolumn{2}{|c|}{ Inv $\times$ UnInv } & \multicolumn{2}{|c|}{ Control x Inv } & \multicolumn{2}{|c|}{ Control $\times$ UnInv } \\
\hline & & & \multicolumn{2}{|c|}{ Involved limb } & \multicolumn{3}{|c|}{$\begin{array}{l}\text { Uninvolved } \\
\text { limb }\end{array}$} & \multirow[b]{2}{*}{$\begin{array}{l}\text { p- } \\
\text { values }\end{array}$} & \multirow[b]{2}{*}{$\begin{array}{l}\text { Effect } \\
\text { size }\end{array}$} & \multirow[b]{2}{*}{$\begin{array}{l}\text { p- } \\
\text { values }\end{array}$} & \multirow[b]{2}{*}{$\begin{array}{l}\text { Effect } \\
\text { size }\end{array}$} \\
\hline & $\begin{array}{l}\text { Mean } \\
\pm \text { SD }\end{array}$ & $\begin{array}{l}\text { Median } \\
\text { [IQR] }\end{array}$ & $\begin{array}{l}\text { Mean } \\
\pm S D\end{array}$ & $\begin{array}{l}\text { Median } \\
\text { [IQR] }\end{array}$ & $\begin{array}{l}\text { Mean } \\
\pm \text { SD }\end{array}$ & $\begin{array}{l}\text { Median } \\
\text { [IQR] }\end{array}$ & $\begin{array}{l}\text { p- } \\
\text { values }\end{array}$ & & & & \\
\hline \multicolumn{12}{|c|}{ Echo-type I † } \\
\hline $\begin{array}{l}\text { Patella } \\
\text { Apex }\end{array}$ & $\begin{array}{l}65.9 \\
\pm 11.0\end{array}$ & $\begin{array}{l}68[60- \\
74]^{* \star *}\end{array}$ & $\begin{array}{l}55.5 \\
\pm 14.3\end{array}$ & $\begin{array}{l}57 \\
{[46-} \\
66]^{\star}\end{array}$ & $\begin{array}{l}54.4 \\
\pm 17.0\end{array}$ & $\begin{array}{l}59[45- \\
66]^{\star \star}\end{array}$ & 0.59 & $<0.01$ & 0.8 & $<0.01$ & 0.7 \\
\hline $\begin{array}{l}\text { Proximal } \\
\text { Tendon }\end{array}$ & $\begin{array}{l}68.0 \\
\pm 11.9\end{array}$ & $\begin{array}{l}72[66- \\
76]\end{array}$ & $\begin{array}{l}61.4 \\
\pm 12.5\end{array}$ & $\begin{array}{l}64 \\
{[57-} \\
70]\end{array}$ & $\begin{array}{l}63.3 \\
\pm 14.6\end{array}$ & $\begin{array}{l}68[56- \\
74]\end{array}$ & 0.21 & 0.01 & 0.5 & 0.19 & 0.3 \\
\hline $\begin{array}{l}\text { Mid } \\
\text { tendon }\end{array}$ & $\begin{array}{l}60.5 \\
\pm 17.3\end{array}$ & $\begin{array}{l}66[50- \\
75]\end{array}$ & $\begin{array}{l}65.0 \\
\pm 12.5\end{array}$ & $\begin{array}{l}68 \\
{[58-} \\
74]\end{array}$ & $\begin{array}{l}66.8 \\
\pm 12.6\end{array}$ & $\begin{array}{l}69[60- \\
76]\end{array}$ & 0.28 & 0.44 & 0.3 & 0.33 & 0.5 \\
\hline $\begin{array}{l}\text { Distal } \\
\text { Tendon }\end{array}$ & $\begin{array}{l}34.0 \\
\pm 18.9\end{array}$ & $\begin{array}{l}30[21- \\
45]^{\star \star \star}\end{array}$ & $\begin{array}{l}57.2 \\
\pm 15.3 \\
\star \star \star *\end{array}$ & $\begin{array}{l}60 \\
{[46-} \\
69]^{*}\end{array}$ & $\begin{array}{l}55.5 \\
\pm 18.6 \\
\star \star \star \star\end{array}$ & $\begin{array}{l}59[42- \\
72]^{\star \star}\end{array}$ & 0.25 & $<0.01$ & 1.5 & $<0.01$ & 1.2 \\
\hline \multicolumn{12}{|c|}{ Echo-type II † } \\
\hline $\begin{array}{l}\text { Patella } \\
\text { Apex }\end{array}$ & $\begin{array}{l}25.8 \\
\pm 6.3\end{array}$ & $\begin{array}{l}24[21- \\
29]^{* \star \star}\end{array}$ & $\begin{array}{l}37.7 \\
\pm 11.4 \\
\star * *\end{array}$ & $\begin{array}{l}37 \\
{[29-} \\
45] \text { * }\end{array}$ & $\begin{array}{l}36.6 \\
\pm 11.7\end{array}$ & $\begin{array}{l}35[28- \\
43]^{\star \star}\end{array}$ & 0.43 & $<0.01$ & 1.1 & $<0.01$ & 1 \\
\hline $\begin{array}{l}\text { Proximal } \\
\text { Tendon }\end{array}$ & $\begin{array}{l}24.6 \\
\pm 7.3\end{array}$ & $\begin{array}{l}25[19- \\
28]^{* \star *}\end{array}$ & $\begin{array}{l}34.4 \\
\pm 10.5\end{array}$ & $\begin{array}{l}33 \\
{[27-} \\
40] \text { * }\end{array}$ & $\begin{array}{l}33.2 \\
\pm 12.5\end{array}$ & $\begin{array}{l}30[25- \\
38]^{\star \star}\end{array}$ & 0.31 & $<0.01$ & 1 & $<0.01$ & 0.7 \\
\hline $\begin{array}{l}\text { Mid } \\
\text { tendon }\end{array}$ & $\begin{array}{l}25.9 \\
\pm 9.1\end{array}$ & $\begin{array}{l}23[20- \\
34]\end{array}$ & $\begin{array}{l}31.3 \\
\pm 10.2\end{array}$ & $\begin{array}{l}29 \\
{[25-} \\
38]\end{array}$ & $\begin{array}{l}29.5 \\
\pm 10.5\end{array}$ & $\begin{array}{l}28 \text { [23- } \\
35]\end{array}$ & 0.20 & 0.03 & 0.5 & 0.17 & 0.4 \\
\hline $\begin{array}{l}\text { Distal } \\
\text { Tendon }\end{array}$ & $\begin{array}{l}27.3 \\
\pm 9.8\end{array}$ & $\begin{array}{l}27[21- \\
33]\end{array}$ & $\begin{array}{l}34.0 \\
\pm 11.6\end{array}$ & $\begin{array}{l}32 \\
{[25-} \\
43]\end{array}$ & $\begin{array}{l}33.6 \\
\pm 11.6\end{array}$ & $\begin{array}{l}31[24- \\
40]\end{array}$ & 0.95 & 0.03 & 0.6 & 0.04 & 0.6 \\
\hline \multicolumn{12}{|c|}{ Echo-type III ${ }^{\dagger} \S$} \\
\hline $\begin{array}{l}\text { Patella } \\
\text { Apex }\end{array}$ & $\begin{array}{l}5.1 \pm \\
7.1\end{array}$ & $2[1-7]$ & $\begin{array}{l}5.0 \pm \\
9.0\end{array}$ & $2[0-5]$ & $\begin{array}{l}6.8 \pm \\
12.0\end{array}$ & $2[0-8]$ & 0.22 & 0.39 & 0 & 0.72 & 0.2 \\
\hline $\begin{array}{l}\text { Proximal } \\
\text { Tendon }\end{array}$ & $\begin{array}{l}4.3 \pm \\
5.2\end{array}$ & $3[1-5]$ & $\begin{array}{l}2.7 \pm \\
5.4\end{array}$ & $1[0-3]$ & $\begin{array}{l}2.3 \pm \\
3.9\end{array}$ & $1[0-3]$ & 0.77 & 0.01 & 0.3 & 0.01 & 0.5 \\
\hline $\begin{array}{l}\text { Mid } \\
\text { tendon }\end{array}$ & $\begin{array}{l}8.7 \pm \\
9.1\end{array}$ & $\begin{array}{l}6[2- \\
13]^{\star * \star}\end{array}$ & $\begin{array}{l}2.4 \pm \\
4.6\end{array}$ & $\underset{*}{1[0-2]}$ & $\begin{array}{l}2.4 \pm \\
4.9\end{array}$ & $1_{\star \star}[0-3]$ & 0.73 & $<0.01$ & 1.1 & $<0.01$ & 1.1 \\
\hline $\begin{array}{l}\text { Distal } \\
\text { Tendon }\end{array}$ & $\begin{array}{l}28.6 \\
\pm 18.2\end{array}$ & $\begin{array}{l}30[16- \\
38]^{* \star *}\end{array}$ & $\begin{array}{l}6.1 \pm \\
8.5^{\text {** }}\end{array}$ & $\underset{*}{3[1-7]}$ & $\begin{array}{l}8.2 \pm \\
11.6 \\
\star \star \star *\end{array}$ & $\begin{array}{l}3[0- \\
10]^{\star *}\end{array}$ & 0.22 & $<0.01$ & 2.0 & $<0.01$ & 1.6 \\
\hline
\end{tabular}




\begin{tabular}{|c|c|c|c|c|c|c|c|c|c|c|c|}
\hline \multirow{3}{*}{$\begin{array}{l}\text { Tendon } \\
\text { quality } \\
\text { (\%) }\end{array}$} & \multirow{2}{*}{\multicolumn{2}{|c|}{ Control $(n=20)$}} & \multicolumn{2}{|c|}{$A C L-D(n=81)$} & & \multicolumn{2}{|c|}{ Inv $\times$ Unlnv } & \multicolumn{2}{|c|}{ Control x Inv } & \multicolumn{2}{|c|}{ Control x UnInv } \\
\hline & & & \multicolumn{2}{|c|}{ Involved limb } & \multicolumn{3}{|c|}{$\begin{array}{l}\text { Uninvolved } \\
\text { limb }\end{array}$} & \multirow[b]{2}{*}{$\begin{array}{l}\text { p- } \\
\text { values }\end{array}$} & \multirow[b]{2}{*}{$\begin{array}{l}\text { Effect } \\
\text { size }\end{array}$} & \multirow[b]{2}{*}{$\begin{array}{l}\text { p- } \\
\text { values }\end{array}$} & \multirow[b]{2}{*}{$\begin{array}{l}\text { Effect } \\
\text { size }\end{array}$} \\
\hline & $\begin{array}{l}\text { Mean } \\
\pm \text { SD }\end{array}$ & $\begin{array}{l}\text { Median } \\
\text { [IQR] }\end{array}$ & $\begin{array}{l}\text { Mean } \\
\pm \text { SD }\end{array}$ & $\begin{array}{l}\text { Median } \\
\text { [IQR] }\end{array}$ & $\begin{array}{l}\text { Mean } \\
\pm \text { SD }\end{array}$ & $\begin{array}{l}\text { Median } \\
\text { [IQR] }\end{array}$ & $\begin{array}{l}\mathrm{p}- \\
\text { values }\end{array}$ & & & & \\
\hline $\begin{array}{l}\text { Patella } \\
\text { Apex }\end{array}$ & $\begin{array}{l}3.1 \pm \\
4.6\end{array}$ & $1[1-3]$ & $\begin{array}{l}1.9 \pm \\
4.7\end{array}$ & $1[0-2]$ & $\begin{array}{l}2.2 \pm \\
3.7\end{array}$ & $1[0-2]$ & 0.45 & 0.04 & 0.3 & 0.12 & 0.2 \\
\hline $\begin{array}{l}\text { Proximal } \\
\text { Tendon }\end{array}$ & $\begin{array}{l}2.8 \pm \\
3.6\end{array}$ & $2[1-4]$ & $\begin{array}{l}1.4 \pm \\
3.7^{\star * *}\end{array}$ & $\underset{*}{0}[0-1]$ & $\begin{array}{l}1.1 \pm \\
2.4 \\
\star \star \star \star\end{array}$ & $\underset{* \star}{0}[0-1]$ & 0.60 & $<0.01$ & 0.4 & $<0.01$ & 0.6 \\
\hline $\begin{array}{l}\text { Mid } \\
\text { tendon }\end{array}$ & $\begin{array}{l}5.0 \pm \\
4.7\end{array}$ & $\underset{\star \star \star}{4[2-7]}$ & $\begin{array}{l}1.3^{ \pm} \\
4.3^{\text {*** }}\end{array}$ & $\underset{*}{0}[0-1]$ & $\begin{array}{l}1.1 \pm \\
2.9 \\
* \star \star \star\end{array}$ & $\underset{* \star}{0}[0-1]$ & 0.95 & $<0.01$ & 0.9 & $<0.01$ & 1.2 \\
\hline $\begin{array}{l}\text { Distal } \\
\text { Tendon }\end{array}$ & $\begin{array}{l}10.1 \\
\pm 6.2\end{array}$ & $\begin{array}{l}11[6- \\
15]^{* * *}\end{array}$ & $\begin{array}{l}2.5 \pm \\
4.4^{\text {*** }}\end{array}$ & ${ }_{*}[0-3]$ & $\begin{array}{l}2.7 \pm \\
3.8 \\
\star \star \star \star\end{array}$ & $1_{* \star}[0-4]$ & 0.76 & $<0.01$ & 1.6 & $<0.01$ & 1.7 \\
\hline \multicolumn{12}{|c|}{ ACL-D = anterior cruciate ligament deficient. } \\
\hline \multicolumn{12}{|c|}{ Inv = involved limb. } \\
\hline \multicolumn{12}{|c|}{ UnInv = Uninvolved limb. } \\
\hline \multicolumn{12}{|c|}{${ }^{\dagger}$ Wilcoxon Signed Ranks Test. } \\
\hline \multicolumn{12}{|c|}{ ‡ Paired Samples Test. } \\
\hline \multicolumn{12}{|c|}{ § Mann-Whitney U. } \\
\hline \multicolumn{12}{|c|}{ ๑ Independent Samples Test (Equal variances assumed). } \\
\hline \multicolumn{12}{|c|}{ * Statistical difference between Involved limb × Controls. } \\
\hline \multicolumn{12}{|c|}{${ }^{\star \star}$ Statistical difference between Uninvolved limb × Controls. } \\
\hline \multicolumn{12}{|c|}{${ }^{\star \star \star}$ Difference between Involved limb × Controls greater than the MDC. } \\
\hline \multicolumn{12}{|c|}{${ }^{\star \star \star \star}$ Difference between Uninvolved limb $\times$ Controls greater than the MDC. } \\
\hline \multicolumn{12}{|c|}{ p-values in bold represent statistical significance. } \\
\hline
\end{tabular}

Comparisons between the patellar tendons of involved and uninvolved limbs of ACL-D with the matching controls tendons yielded statistically significant differences in several variables of tendon quality (Table 2). However, only a few of these variables displayed differences between groups which were greater than the MDC (Table 2; FIGURE 4) [17].

ACL-D displayed similar echo-pattern distribution in all the areas within the tendon in contrast to control tendons that presented a completely different appearance in the proximal half of the tendon in comparison to the distal half. However, in both limbs, ACL-D displayed more echo-type II associated to less echo-type I at the patellar apex and at the proximal tendon, and same or less echo-types III and IV in all areas than the control tendons (Table 2; FIGURE 4).

Similar patellar tendon characteristics were observed in the 12 ACL-D knees with anterior pain (AKP), in the 41 ACL-D with pain in a different area of the knee (other knee pain: OKP) and in the 109 knees without pain (WP) (Table 3). There were no 
significant differences in VAS $(P=0.55)$ and knee extensor strength $(P=0.35)$ between participants with AKP (VAS: 3.0

[2.3-4.5]; Ext PT\%BW: $203.4 \pm 68.2 \%$ ) and other knee pain (VAS: 4.0 [2.0-5.0]; Ext PT\%BW: $225.3 \pm 69.2 \%$ ). Conversely, the 96 out of 109 asymptomatic knees of ACL-D participants who performed the isokinetic test displayed significantly higher knee extensor strength $(277.2 \pm 57.3 \%)$ than the two groups with pain (AKP $\times$ WP: ES $=1.3 ; P<0.01 ; \mathrm{OKP} \times \mathrm{WP}: \mathrm{ES}=0.9 ; P<$ $0.01)$. 
Table 3

Tendon quality and comparisons results among ACL-D with AKP, other knee pain, and without pain.

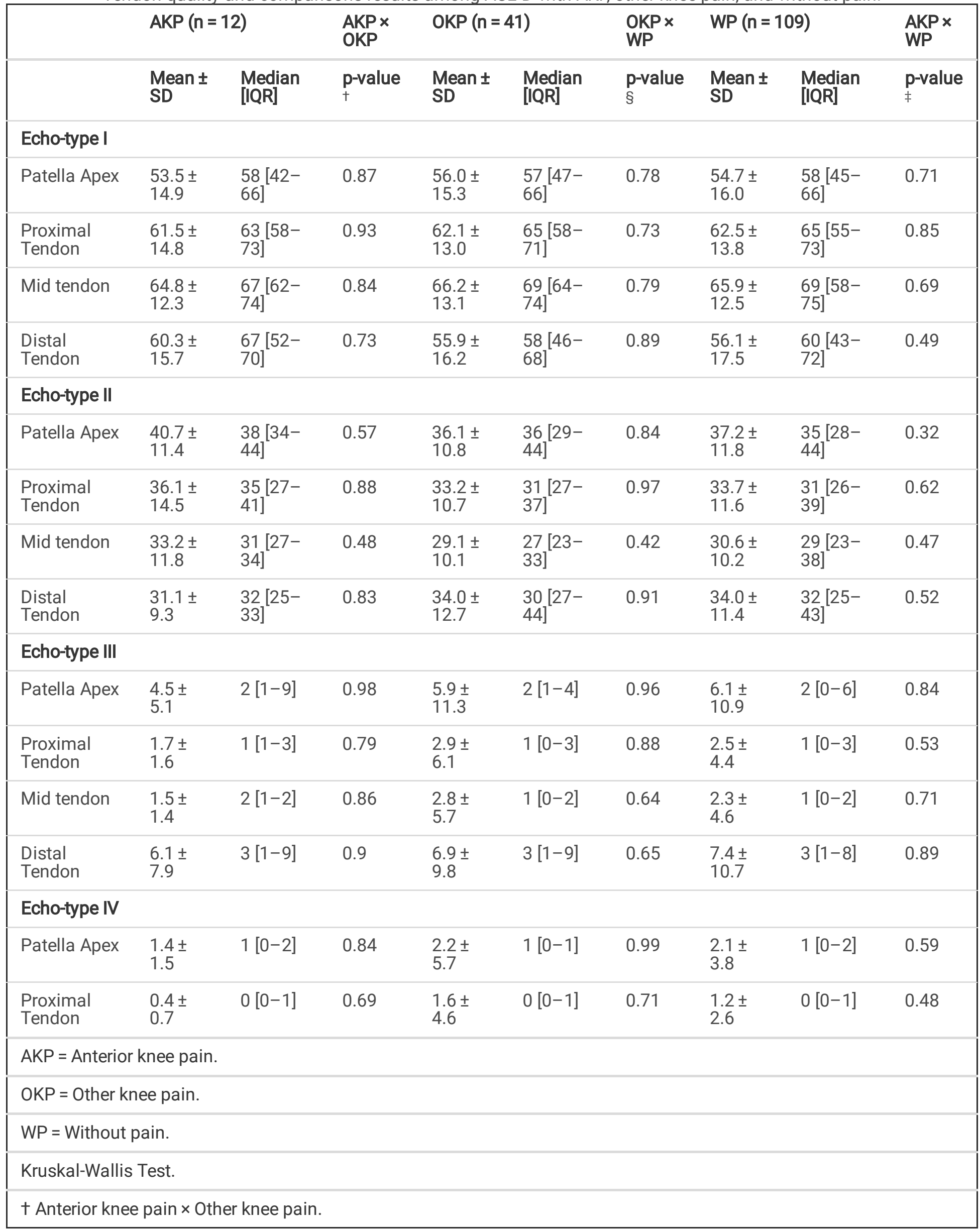




\begin{tabular}{|c|c|c|c|c|c|c|c|c|c|}
\hline \multirow[b]{2}{*}{ Mid tendon } & \multicolumn{2}{|c|}{$\operatorname{AKP}(n=12)$} & \multirow{2}{*}{$\begin{array}{l}\begin{array}{l}\text { AKP } x \\
\text { OKP }\end{array} \\
0.66\end{array}$} & \multicolumn{2}{|c|}{ OKP $(n=41)$} & \multirow{2}{*}{$\begin{array}{l}\begin{array}{l}\text { OKP } x \\
\text { WP }\end{array} \\
0.42\end{array}$} & \multicolumn{2}{|c|}{ WP $(n=109)$} & \multirow{2}{*}{$\begin{array}{l}\begin{array}{l}\text { AKP } \\
\text { WP }\end{array} \\
0.76\end{array}$} \\
\hline & $\begin{array}{l}0.5 \pm \\
0.7\end{array}$ & $0[0-1]$ & & $\begin{array}{l}1.9 \pm \\
5.8\end{array}$ & $1[0-1]$ & & $\begin{array}{l}1.1 \pm \\
2.6\end{array}$ & $0[0-1]$ & \\
\hline $\begin{array}{l}\text { Distal } \\
\text { Tendon }\end{array}$ & $\begin{array}{l}2.4 \pm \\
3.3\end{array}$ & $2[0-3]$ & 0.8 & $\begin{array}{l}3.0 \pm \\
5.5\end{array}$ & $1[0-3]$ & 0.53 & $\begin{array}{l}2.5 \pm \\
3.6\end{array}$ & $1[0-4]$ & 0.74 \\
\hline \multicolumn{10}{|c|}{ AKP = Anterior knee pain. } \\
\hline \multicolumn{10}{|c|}{ OKP = Other knee pain. } \\
\hline \multicolumn{10}{|c|}{ WP = Without pain. } \\
\hline \multicolumn{10}{|c|}{ Kruskal-Wallis Test. } \\
\hline
\end{tabular}

$\ddagger$ Anterior knee pain $\times$ Without pain.

$\S$ Other knee pain $\times$ Without pain.

Detailed information about the associations between patellar tendon quality and the variables of: VAS, knee extensor strength, and the time from the ACL injury are presented in Table 4. There were no meaningful associations between the amount of knee pain on the assessment day with any of the studied variables of tendon quality. However, there was a significant negative association between knee pain and knee extensor isokinetic strength $(\mathrm{R}=-0.41 ; P<0.01)$. Unsurprisingly, the greater the knee pain the less the knee extensor muscles were able to generate torque. In general, greater time from ACL injury was positively associated with echo-types I and II, and negatively associated with echo-types III and IV (Table 4). 
Table 4

Values of the associations between variables of tendon quality and knee symptoms ( $n=162)$.

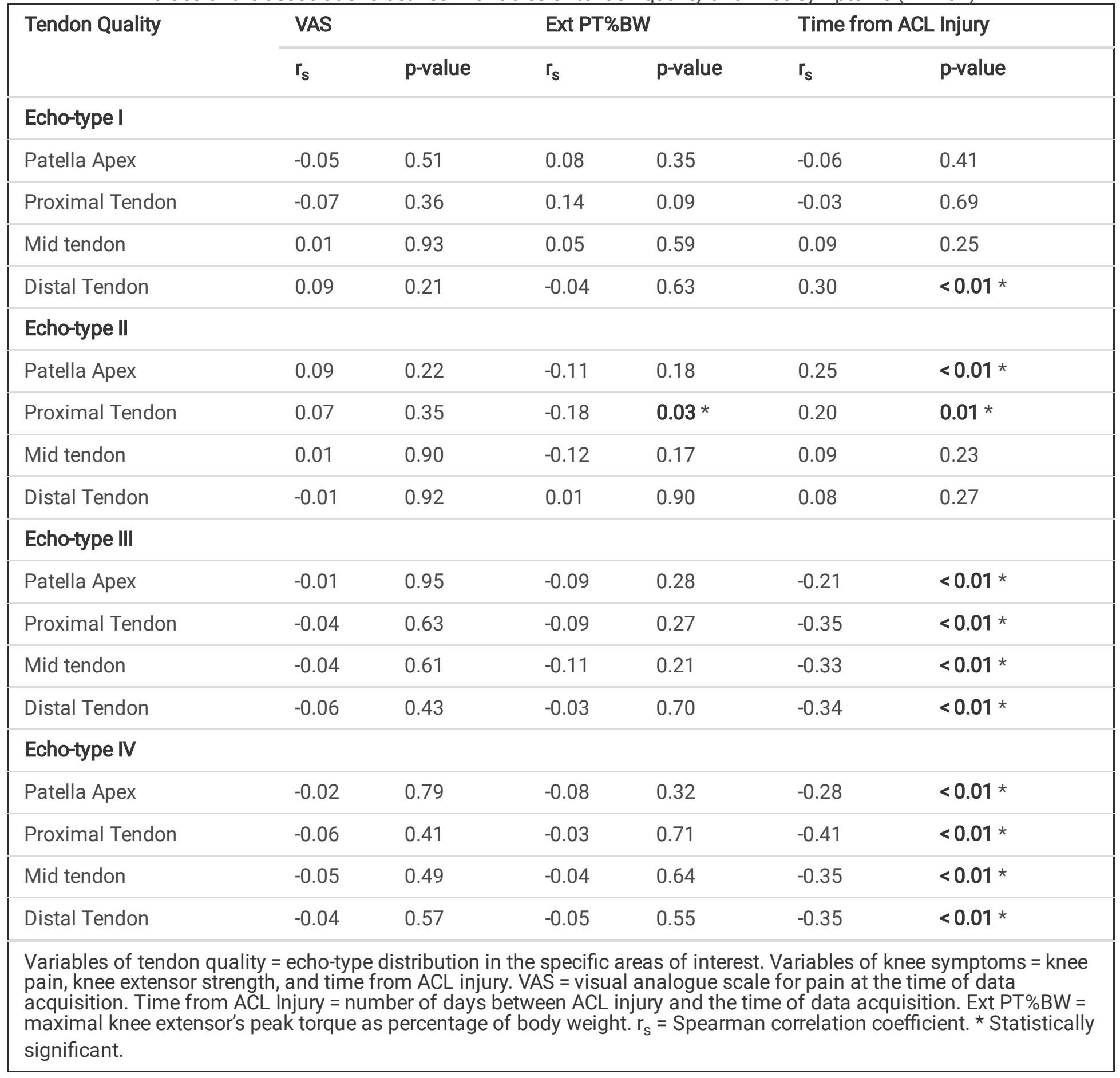

\section{Discussion}

To the authors' knowledge, this is the first study to assess patellar tendon quality in ACL-D athletes. The study is also novel in that it is one of the few studies using UTC to characterize the quality in the whole tendon length (apex, proximal, mid, and distal).

\section{There is no difference in tendon quality between limbs of ACL-D athletes}

The initial hypotheses that the involved patellar tendon of ACL-D athletes would present lower quality on the UTC scan in comparison to the uninvolved tendon was rejected. Although more than half of the ACL-D athletes presented some pain in 
the knee, and displayed significantly lower knee extensor strength in the involved in comparison to the uninvolved limb, the distribution of echo-types characterizing the tendon was similar in all studied areas of the tendons. The characteristics of both ACL-D tendons in the current study are comparable with previous values described in the literature for healthy (approximately $68 \%, 32 \%$, nearly $1 \%$, and $0 \%$, for echo-types I, II, III and IV respectively), and asymptomatic (approximately $60 \%, 37 \%, 2 \%, 1 \%$, for echo-types I, II, III and IV respectively) patellar tendons [21]. van Ark et al. (2019) tested the reliability of UTC imaging in symptomatic and asymptomatic patellar tendons using window setting of 25 in the analysis, which provides less detailed depiction of tendon structure compared to 17 used in the current study [16]. van Ark et al. (2019) and Rabello et al. (2019) also adopted a different methodology to delineate the contours using the proximal $2 \mathrm{~cm}$ only, disregarding the distal portions of the tendon $[16,21]$. The characteristics of their asymptomatic group though were very similar to the values displayed in the mid-tendon of ACL-D tendons in the current study [16]. These differences notwithstanding, the small differences in outcomes between studies are mostly within the values of the MDC that have been previously described for this device and tendon $[16,17,21]$.

\section{ACL-D displayed slightly worse proximal tendon than the controls}

The second hypothesis that both patellar tendons of ACL-D would display lower UTC scan quality than the controls was partially rejected. As hypothesized, ACL-D participants displayed more echo-type II and less echo-type I than the controls at the patellar apex and proximal tendon, in the involved and uninvolved limbs, suggesting that both ACL-D tendons were slightly worse than the active asymptomatic controls at the proximal half of the patellar tendon. Likely, the fact that ACL-D participants were athletes of a variety of sports but were experiencing a period of inactivity due to the ACL injury might explain such findings. It has been previously proposed that a period of disuse could cause tendon deterioration and reduced tendon stiffness [27]. On the other hand, ACL-D participants displayed smaller proportion of echo-types III and IV, which could represent better tendon quality than the controls, refuting the initial hypotheses. Unexpectedly, the distal half of the control tendons displayed completely different properties than its proximal half, with much higher amounts of echotypes III and IV, i.e. worse tendon quality, than previously described [17, 28]. Kongsgaard et al (2007) noted that different areas of the patellar tendon might respond differently to the same strengthening stimulus [29]. Potentially, these findings may relate to the fact that the control athletes were currently in-season, and exposed to high external loads which may affect the patellar tendon and hence may explain the low quality in the distal portion of the tendon [30, 31]. It has been previously observed in the patellar tendon of volleyball players [32] and in healthy Achilles tendons of Australian football players' [20] a transient adaptation in echo-types distribution in response to loads. To the best of our knowledge, only two studies have explored the distal portion of the patellar tendon $[17,28]$. Interestingly, most of the studies using UTC to track adaptations in tendons have described interexchange between echo-types I and II, or between echo-types III and IV [20, 22, 32]. However, the control group of the current study displayed less echo-type I at the expense of increasing the proportion of echo-types III and IV. Whether training load could be the cause of this remains to be answered, as well whether this adaptation is temporary or permanent. It would be useful to further investigate, longitudinally, if the quality observed in the control tendons in this study is a common phenomenon in athletes of kicking sports, if it reflects a transient adaptation due to the intense loads of in-season, or yet if athletes of different sports with different demands would present different tendon features.

\section{No differences in tendon quality regardless of pain presence and location}

The third hypothesis that ACL-D with AKP would present lower tendon quality in comparison to ACL-D with other knee pain, and ACL-D without knee pain was also refuted. Knee pain, regardless of location, might have an impact in the ability to generate knee extension torque, however, the presence of pain was not enough to differentiate the quality of these symptomatic or asymptomatic patellar tendons. Previous studies have also found that the presence and severity of pain did not reflect the tendon quality observed in UTC scans. Docking et al (2018) assessed 152 patellar tendons of elite male Australian football players at the preseason, to observe that even though there were differences in the cross-sectional area and in the proportion of echo-types I + II when comparing athletes with and without pathology, symptoms or previous

Page $13 / 22$ 
history, there was no association between tendon quality and presence, development or severity of symptoms in the tendon [33].

\section{No association between knee pain, knee extensor strength and tendon quality even though, tendon quality seems to improve with longer time from ACL injury.}

Although abnormal tendons on ultrasound have a fivefold increased likelihood of developing tendinopathy and becoming symptomatic [34], results of the current study pointed to a lack of association between the amount of knee pain and any of the tendon quality variables explored [33]. From the 23 patellar tendons of ACL-D participants that displayed a clear disorganized area in the UTC scan, 5 had history of patellar tendinopathy, but only 2 of these tendons were associated with AKP. Such observations suggest that other structures in the knee, rather than the patellar tendon, might be causing the AKP, and that signs of tendon disorganization in the UTC scans did not reflect symptoms. It seems that UTC was useful to detect altered echo signs in tendons, but it was not sensitive enough to differentiate painful tendons.

In agreement with our results of knee extensor strength, ACL-D patients often present altered patterns of muscle activation $[9,35]$, and reduced knee function in the involved limb $[9,24,35,36]$. A recent systematic review of quadriceps activation in ACL-D, ACL-reconstructed, and AKP patients showed that individuals who suffer from AKP seem to have even more compromised neuromuscular deficits than people with ligament injuries $[9,11]$.

Moreover, the longer the time from ACL injury the better the tendon quality became. We speculate that the longer the time from the injury, longer period the injured knee would have to settle from the initial symptoms of pain, and swelling, leading to improved function. Kongsgaard et al (2007) [29], Magnusson et al (2008) [27], and Kjær et al (2009) [37] believe that tendon adapts to changes in load, and proposed that offloading the lower limb could lead to degradation of the mechanical properties of the tendon. However, reintroducing load and implementing a resistive training program could positively affect the tendon properties and increase load capacity.

\section{Conclusions}

One of the main findings of the current study is that the involved and uninvolved patellar tendons of ACL-D displayed similar echo-pattern distribution, suggesting that a unilateral ACL injury did not influence the quality of the tendons of either knee. Interestingly, different parts of the tendon might present with different tendon characteristics.

ACL-D with knee pain generated significant less knee extensor strength than asymptomatic knees. However, regardless of presence and location of pain, the patellar tendons of ACL-D athletes displayed similar properties in all variables characterizing tendon quality.

Furthermore, the amount of knee pain and knee extensor strength did not display any significant association with any of the variables of tendon quality, suggesting that the patellar tendon is rarely the cause of AKP in ACL-D athletes. Nevertheless, some weak to moderate associations suggest that tendon quality improved as time from injury increased.

\section{Key Points}

\section{Findings}

- There was no difference in the patellar tendon quality of the involved and uninvolved limbs of ACL-D athletes.

- Different areas of the patellar tendon may present different characteristics in response to load, as evidenced by the worse quality displayed at the distal tendon of the asymptomatic but highly active control group.

- The proximal half of ACL-D tendons presented worse quality than the controls. 
- There was no difference in the quality of ACL-D tendons regardless of pain presence and location, even though knee pain was associated to reduced knee extensor strength.

- Patellar tendon seems to be rarely the cause of AKP in ACL-D athletes.

- The patellar tendon quality seemed better in ACL-D athletes with longer time from ACL injury.

\section{Implications}

The lack of differences between the involved and uninvolved limbs of athletes sustaining a unilateral ACL injury suggests that the patellar tendon of the uninvolved limb can be used as comparison for future longitudinal studies.

It was not possible to differentiate the patellar tendons of ACL-D athletes with anterior knee pain, from ACL-D athletes with other knee pain or without pain, suggesting that either the patellar tendon is rarely the source of knee pain or that the UTC is not sensible enough to highlight such differences.

It would be interesting to further investigate whether the different characteristics within the patellar tendon is related to training loads or sport specific.

\section{Caution}

This study is limited by the fact that the number of physiotherapy sessions before the data acquisition was not considered. The amount of rehabilitation could have positively affected the knee condition and consequently the patellar tendon quality. It is also limited by the size, sport (only football), as well the physical activity level of the participants of the control group at the time of data acquisition. Such facts might represent bias in the patellar tendon quality of the controls. Additionally, in order to compare studies and avoid misleading information, it is important to highlight that the pre-setting of window 17 was adopted in the UTC imaging software to analyze the characteristics of the patellar tendons of ACL-D athletes and controls in the current study.

\section{List Of Abbreviations}

ACL: anterior cruciate ligament

ACL-D: anterior cruciate ligament deficient

UTC: ultrasound tissue characterization

AKP: anterior knee pain

ROM: range of motion

VAS: Visual analogue scale

BW: body weight

MRI: magnetic resonance imaging

MDC: minimal detectable change

$\mathrm{n}$ : group sample size

$\mathrm{N}$ : total sample size

Nm: Newton meters 
Kg: Kilogram

SD: standard deviation

IQR: interquartile

Min: minimum

Max: maximum

g: Hedge's coefficient

ES: effect size

$r_{s}$ : Spearman correlation coefficient

P. p-value

OKP: Other knee pain

WP: without pain

Inv: Involved limb

UnInv: Uninvolved limb

ExtPT\%BW: maximal knee extensor's peak torque as percentage of body weight

\section{Declarations}

\section{Ethics Approval and consent to participate}

The study protocol was approved by ethical committee of the Anti-Doping Laboratory Qatar Research Office (2017000227).

Written informed consent was obtained from each participant.

\section{Consent for publication}

Not applicable.

\section{Availability of data and material}

The datasets used and analyzed during the current study are available from the correspondent author on reasonable request.

\section{Competing interests}

The authors of this manuscript declare that they have no competing interests in any matter included in this manuscript.

\section{Funding}

The funding for the publication of this article in open access format was provided by the Qatar National Library and the University of Jyväskylä. 
C.S.P. was responsible for the conception and design of the study, acquisition, analysis, and interpretation of data, in drafting the manuscript, and agreed to be accountable for all aspects of this research.

J.K. made substantial contributions to the acquisition, analysis and interpretation of data, reviewed it critically for important intellectual content, and agreed to be accountable for all aspects of this research.

S.M. made substantial contributions to the acquisition, analysis and interpretation of data, reviewed it critically for important intellectual content, and agreed to be accountable for all aspects of this research.

R. W. made substantial contributions to conception and design of this study, reviewed it critically for important intellectual content, gave final approval of the version to be published, and agreed to be accountable for all aspects of this research.

T.F. made substantial contributions to conception and design of this study, reviewed it critically for important intellectual content, gave final approval of the version to be published, and agreed to be accountable for all aspects of this research.

\section{Acknowledgments}

Authors would like to thank Antonio Carlos Pereira, Argyro Kotsifaki, Bart Sas, Caio Augusto Soncino Pereira, Carla de Paula, Cleuza Soncino Pereira, Diane Slater, Dustin Maree, Erik Witvrouw, Evan Jeanguyot, Jaleleddine Belhaj, Martina Jakob Emeršič, Mayolo Camacho, Mansour Otayek, Mirna Anadani, Luka Pereira Kuitunen, Oana Gheorghita, Paul Read, Riadh Miladi, Sami Kuitunen, and Vasileios Sideris for all the contribution and support.

\section{Author's information}

C.S.P. Physical therapist, MSc, Rehabilitation Department - ASPETAR Orthopedic and Sports hospital, Doha, Qatar, and PhD candidate, Faculty of Sport and Health Sciences, Biology of Physical Activity, Neuromuscular Research Center, University of Jyväskylä, Jyväskylä, Finland. carla.pereira@aspetar.com

J.K. Physical therapist assistant, Rehabilitation Department - ASPETAR Orthopedic and Sports hospital, Doha, Qatar. jasenko.klauznicer@aspetar.com

S.M. Physical therapist, PhD, former clinical researcher scientist - ASPETAR Orthopedic and Sports hospital, and Senior Lecturer at the Department of Physical Therapy \& Rehabilitation Science, College of Health Sciences, Qatar University, Doha, Qatar. macca@live.ie

R. W. Assistant Director of Clinical Projects \& Quality, Physical therapist, PhD, Rehabilitation Department - ASPETAR Orthopedic and Sports hospital, Doha, Qatar. rodney.whiteley@aspetar.com

T.F. Professor of Kinesiology, PhD, Faculty of Sport and Health Sciences, Biology of Physical Activity, Neuromuscular Research Center, University of Jyväskylä, Jyväskylä, Finland. taija.fınni@jyu.fı

\section{References}

1. Musahl V, Karlsson J. Anterior cruciate ligament tear. N Engl J Med. 2019;380:2341-8.

2. Grindem H, Eitzen I, Engebretsen L, Snyder-Mackler L, Risberg MA. Nonsurgical or surgical treatment of ACL injuries: Knee function, sports participation, and knee reinjury: The Delaware-Oslo ACL cohort study. J Bone Jt Surg - Am Vol. 2014;96:1233-41.

3. De Carlo M, Armstrong B. Rehabilitation of the knee following sports injury. Clin Sports Med. 2010;29:81-106. doi:10.1016/j.csm.2009.09.004. 
4. Dunn WR, Spindler KP, Amendola A, Andrish JT, Kaeding CC, Marx RG, et al. Which preoperative factors, including bone bruise, are associated with knee pain/ symptoms at index anterior cruciate ligament reconstruction (ACLR)?: A multicenter orthopaedic outcomes network (MOON) aclr cohort study. Am J Sports Med. 2010;38:1778-87.

5. Weiss K. Biomechanics Associated with Patellofemoral Pain and ACL Injuries in Sports. Sport Med. 2015;45:1325-37.

6. Biedert RM, Sanchis-alfonso V. Sources of anterior knee pain. 2002;21:335-47.

7. Christian SR, Anderson MB, Workman R, Conway WF, Pope TL. Imaging of Anterior Knee Pain. 2006;25:681-702.

8. Eitzen I, Holm I, Risberg MA. Preoperative quadriceps strength is a significant predictor of knee function two years after anterior cruciate ligament reconstruction. Br J Sports Med. 2009;43:371-6.

9. Hart JM, Pietrosimone B, Hertel J, Ingersoll CD. Quadriceps activation following knee injuries: a systematic review. J Athl Train. 2010;45:87-97. doi:10.4085/1062-6050-45.1.87.

10. Delaloye JR, Murar J, Vieira TD, Franck F, Pioger C, Helfer L, et al. Knee Extension Deficit in the Early Postoperative Period Predisposes to Cyclops Syndrome After Anterior Cruciate Ligament Reconstruction: A Risk Factor Analysis in 3633 Patients From the SANTI Study Group Database. Am J Sports Med. 2020;:1-8. doi:10.1177/0363546519897064.

11. Kim S, Kim D, Park J. Knee Joint and Quadriceps Dysfunction in Individuals With Anterior Knee Pain, Anterior Cruciate Ligament Reconstruction, and Meniscus Surgery: A Cross-Sectional Study. J Sport Rehabil. 2020;April:1-8.

12. Heijne A, Ang BO, Werner S. Predictive factors for 12-month outcome after anterior cruciate ligament reconstruction. Scand J Med Sci Sports. 2009;19:842-9. doi:10.1111/j.1600-0838.2008.00852.x.

13. Månsson 0 , Kartus J, Sernert N. Pre-operative factors predicting good outcome in terms of health-related quality of life after ACL reconstruction. Scand J Med Sci Sport. 2013;23:15-22.

14. van Schie H, de Vos R, de Jonge S, Bakker E, Heijboer M, Verhaar J, et al. Ultrasonographic tissue characterisation of human Achilles tendons: quantification of tendon structure through a novel non-invasive approach. Br J Sports Med. 2010;44:1153-9.

15. van Schie H, Docking S, Daffy J, Praet S, Rosengarten S, Cook J. Ultrasound tissue characterisation, an innovative technique for injury-prevention and monitoring of tendinopathy. Br J Sport Med. 2013;47:14.

16. van Ark M, Rabello LM, Hoevenaars D, Meijerink J, van Gelderen N, Zwerver J, et al. Inter - and intra - rater reliability of ultrasound tissue characterization (UTC) in patellar tendons. Scand J Med Sci Sport. 2019;29 January:1205-11.

17. Pereira CS, Santos RCG, Whiteley R, Finni T. Reliability and methodology of quantitative assessment of harvested and unharvested patellar tendons of ACL injured athletes using ultrasound tissue characterization. 2019;:1-13.

18. van Schie H, Bakker E, Jonker A, van Weeren P. Computerized ultrasonographic tissue characterization of equine superficial digital flexor tendons by means of stability quantification of echo patterns in contiguous transverse ultrasonographic images. Am J Vet Res. 2003;64:366-75.

19. Rabello LM, Dams OC, van den Akker-Scheek I, Zwerver J, O'Neill S. Substantiating the Use of Ultrasound Tissue Characterization in the Analysis of Tendon Structure. Clin J Sport Med. 2019;00:1.

20. Rosengarten SD, Cook JL, Bryant AL, Cordy JT, Daffy J, Docking SI. Australian football players' Achilles tendons respond to game loads within 2 days: an ultrasound tissue characterisation (UTC) study. Br J Sports Med. 2014;:1-6. doi:10.1136/bjsports-2013-092713.

21. Rabello LM, van den Akker-Scheek I, Kuipers IF, Diercks RL, Brink MS, Zwerver J. Bilateral changes in tendon structure of patients diagnosed with unilateral insertional or midportion achilles tendinopathy or patellar tendinopathy. Knee Surgery Sport Traumatol Arthrosc. 2019. doi:10.1007/s00167-019-05495-2.

22. Esmaeili A, Stewart AM, Hopkins WG, Elias GP, Aughey RJ. Effects of Training Load and Leg Dominance on Achilles and Patellar Tendon Structure. 2017;:122-6.

23. Anderson MJ, Browning WM, Urband CE, Kluczynski MA, Bisson LJ. A Systematic Summary of Systematic Reviews on the Topic of the Anterior Cruciate Ligament. Orthop J Sport Med. 2016;4:2325967116634074. doi:10.1177/2325967116634074.

Page 18/22 
24. Logerstedt DS, Snyder-mackler MAL, Ritter SRC, Axe MJ, Godges JJ, Fitzgerald GK, et al. Knee Stability and Movement Coordination Impairments: Knee Ligament Sprain Clinical Practice Guidelines Linked to the International Classification of Functioning, Disability, and Health from the Orthopaedic Section of the American Physical Therapy Asso. 2010.

25. Flandry F, Hunt JP, Terry GC, Hughston JC. Analysis of subjective knee complaints using visual analog scales. Am J Sports Med. 1991;19:112-8.

26. Cohen J. Statistical power analysis for the behavioral sciences. 2nd Editio. 2003.

27. Magnusson SP, Narici MV, Maganaris CN, Kjaer M. Human tendon behaviour and adaptation. in vivo. 2008;1:71-81.

28. Hernández G, Domínguez D, Moreno J, Til L, Capdevila L. Patellar tendon analysis by ultrasound tissue characterization; comparison between professional and amateur basketball players. Asymptomatic versus symptomatic. Apunt Med l'Esport. 2017;52:45-52.

29. Kongsgaard M, Reitelseder S, Pedersen TG, Holm L, Aagaard P, Kjaer M. Region specific patellar tendon hypertrophy in humans following resistance training. 2007;:111-21.

30. Bode G, Hammer T, Karvouniaris N, Feucht MJ, Konstantinidis L, Südkamp NP, et al. Patellar tendinopathy in young elite soccer- clinical and sonographical analysis of a German elite soccer academy. BMC Musculoskelet Disord. 2017;18:1-7.

31. Flores DV, Gómez CM, Pathria MN. Layered approach to the anterior knee: Normal anatomy and disorders associated with anterior knee pain. Radiographics. 2018;38:2069-101.

32. Rabello LM, Zwerver J, Stewart RE, van den Akker-Scheek I, Brink MS. Patellar tendon structure responds to load over a 7-week preseason in elite male volleyball players. Scand J Med Sci Sport. 2019;29:992-9.

33. Docking SI, Girdwood MA, Cook J, Fortington LV, Rio E. Reduced Levels of Aligned Fibrillar Structure Are Not Associated With Achilles and Patellar Tendon Symptoms. Clin J Sport Med. 2018;00:1. doi:10.1097/JSM.0000000000000644.

34. McAuliffe S, McCreesh K, Culloty F, Purtill H, O'Sullivan K. Can ultrasound imaging predict the development of Achilles and patellar tendinopathy? A systematic review and meta-analysis. Br J Sports Med. 2016;50:1516-23.

35. Ingersoll CD, Grindstaff TL, Pietrosimone BG, Hart JM. Neuromuscular Consequences of Anterior Cruciate Ligament Injury. Clin Sports Med. 2008;27:383-404.

36. Ageberg E. Consequences of a ligament injury on neuromuscular function and relevance to rehabilitation - using the anterior cruciate ligament- injured knee as model. 2002;12:205-12.

37. Kjær M, Langberg H, Heinemeier K, Bayer ML, Hansen M, Holm L, et al. From mechanical loading to collagen synthesis, structural changes and function in human tendon. Scand J Med Sci Sport. 2009;19:500-10.

\section{Figures}




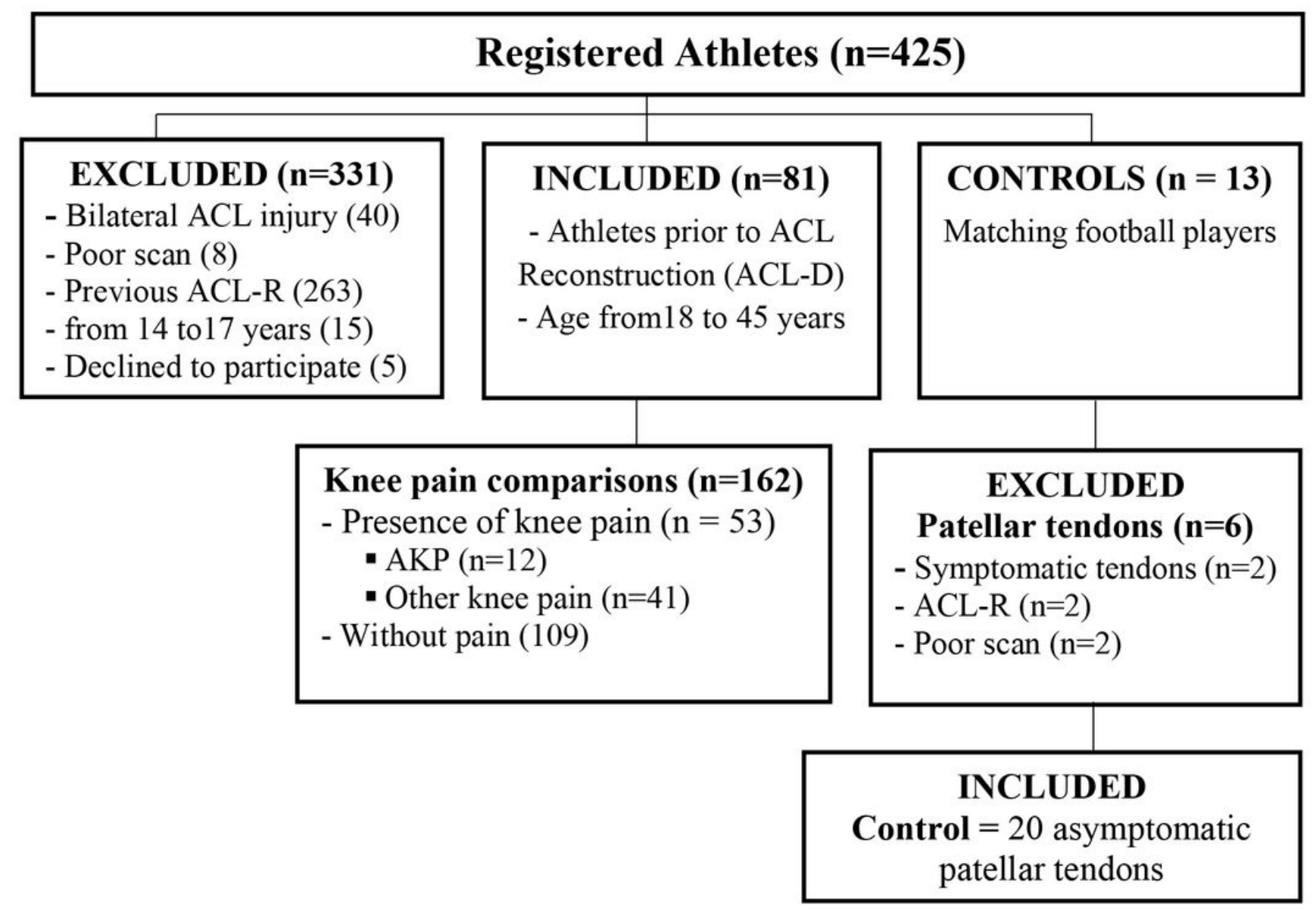

Figure 1

Flow diagram of the participant inclusion and sample sizes for the different studied groups.
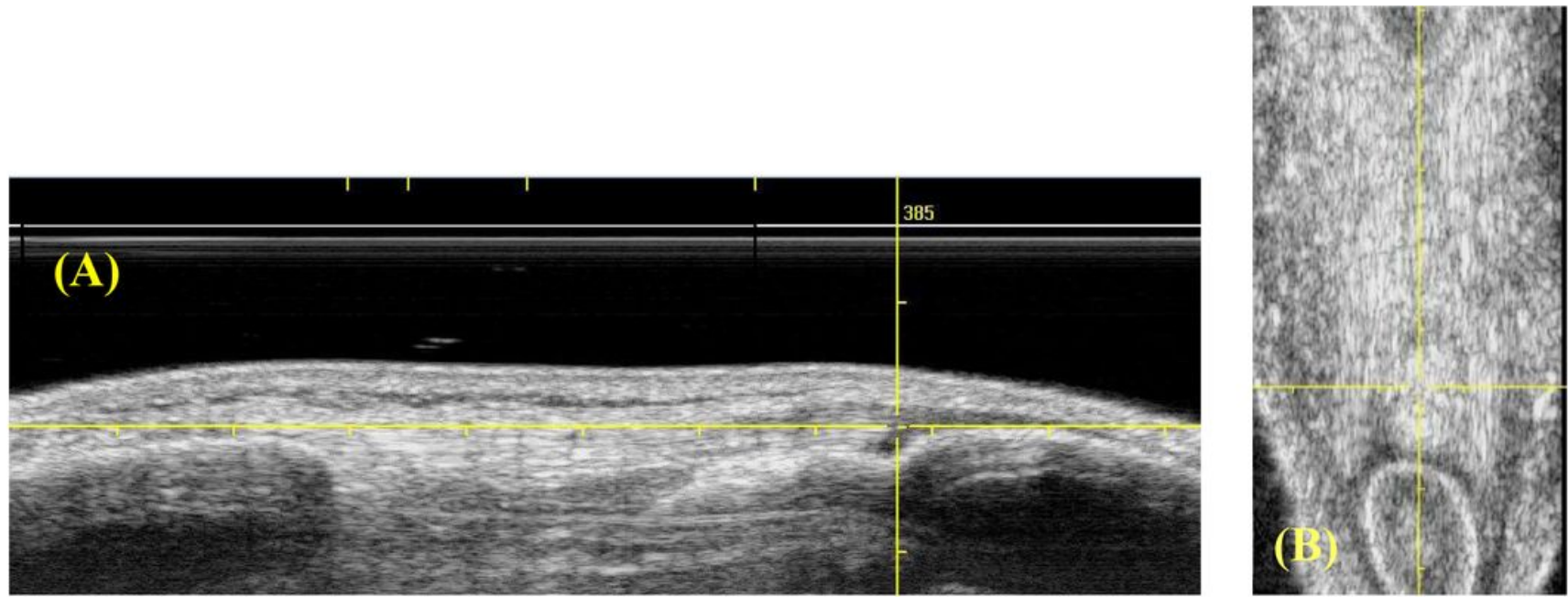

Figure 2 
Example of UTC scans that met the inclusion criteria. (A) Yellow reference line used to check the horizontal alignment in the sagittal view. (B) Yellow reference line used to check the vertical alignment in the frontal view.
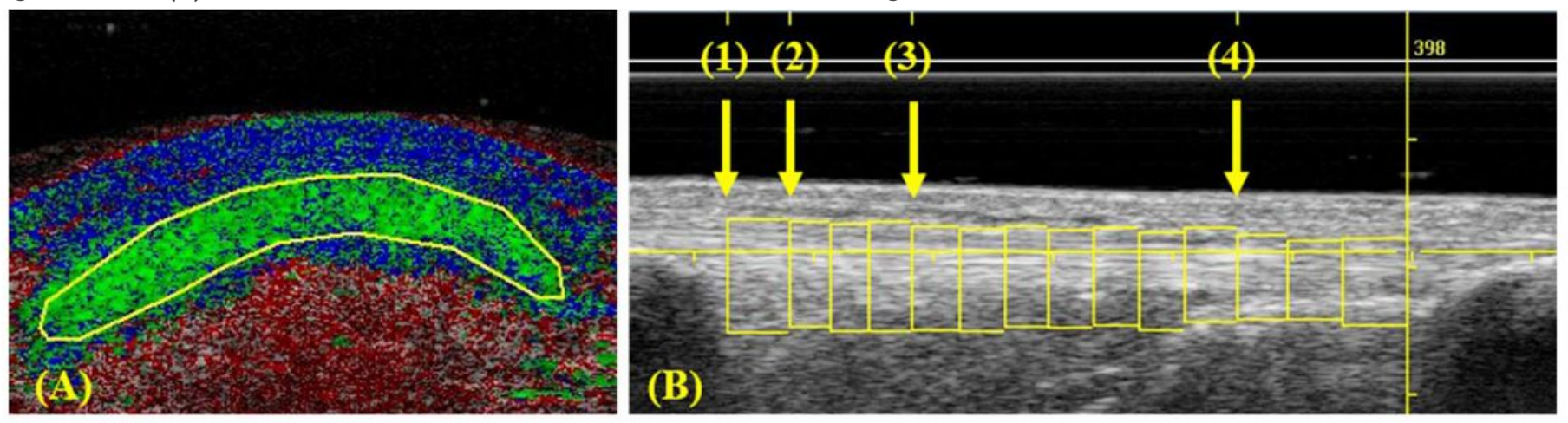

\section{Figure 3}

Patellar tendon on UTC images. (A) Transversal view. (B) Sagittal view illustrating the studied areas. (A) Transverse view of UTC color coded image showing a manually traced contour to delineate the area of the patellar tendon. (B) Sagittal view of the patellar tendon illustrating all the contours traced in the whole length of the patellar tendon. Yellow arrows identify the four levels of the tendon (1-4) where the proportion of echo-types were calculated to sub-characterize the tendon quality. (1) Patellar apex $=$ first image after the patellar apex disappears in the transverse view. (2) Proximal tendon $=a t 0.5 \mathrm{~cm}$ from patellar apex. (3) Mid tendon = at $1.5 \mathrm{~cm}$ from patellar apex. (4) Distal tendon $=$ at $75 \%$ of the tendon length. 


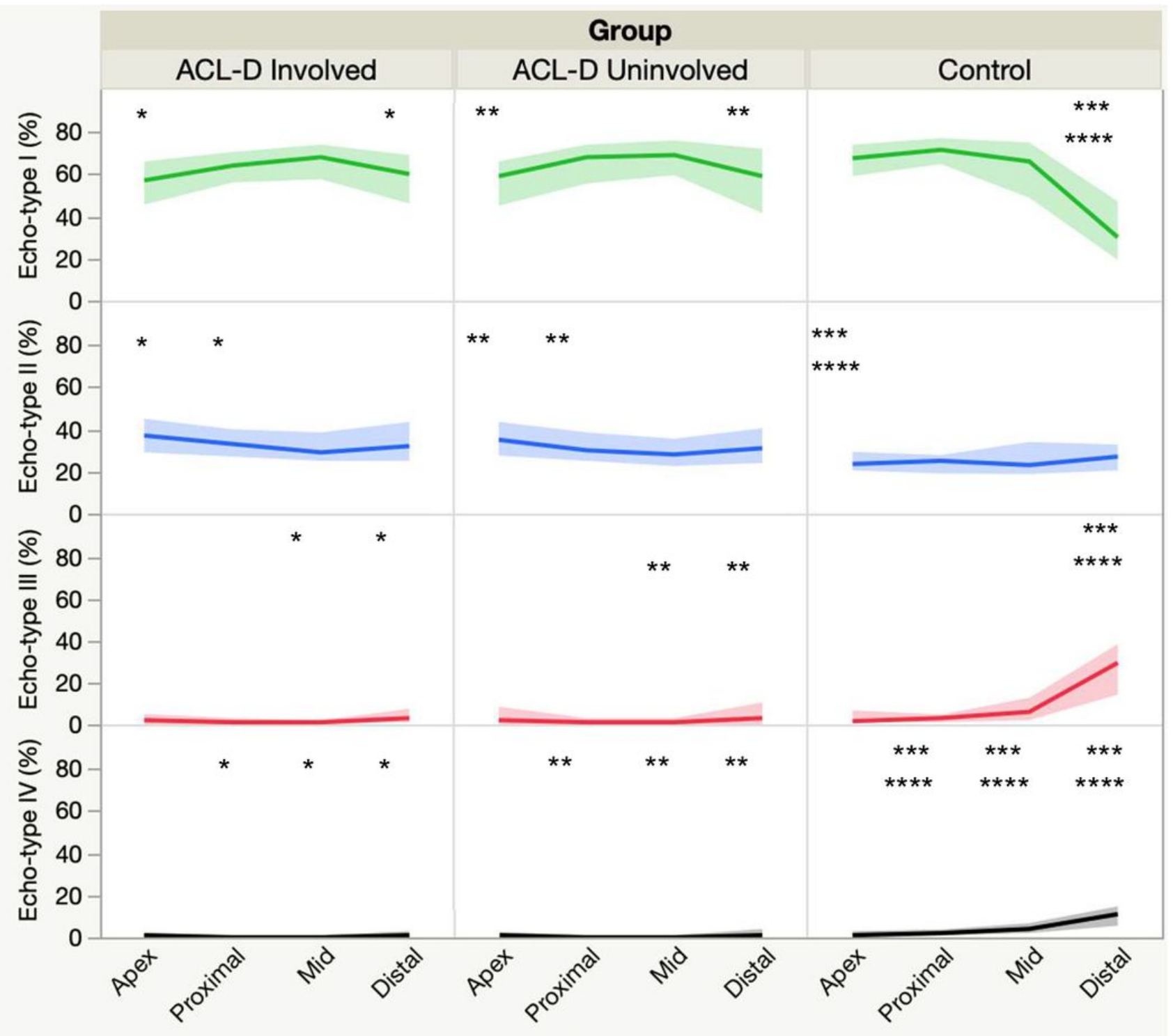

Patellar tendon areas

\section{Figure 4}

Characterization of the specific areas within the patellar tendons of ACL-D ( $n=81)$ and controls $(n=20)$. Darker line $=$ Median of the distribution of echo-types I, II, III and IV. Colorful shade = upper and lower quartile of the distribution of echo-types I, II, III and IV. ACL-D = anterior cruciate ligament deficient. * Statistical difference between Involved limb $\times$ Controls. ** Statistical difference between Uninvolved limb $\times$ Controls. ${ }^{\star} * \star$ Statistical difference greater than the MDC between ACL-D

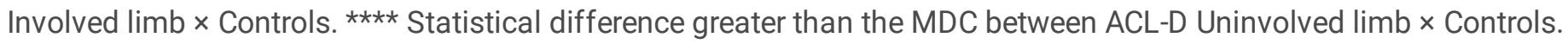

